Forum Math. 10 (1998), 751-773

\title{
Collineations of smooth stable planes
}

\author{
Richard Bödi \\ (Communicated by Karl H. Hofmann)
}

\begin{abstract}
Smooth stable planes have been introduced in [4]. We show that every continuous collineation between two smooth stable planes is in fact a smooth collineation. This implies that the group $\Gamma$ of all continuous collineations of a smooth stable plane is a Lie transformation group on both the set $P$ of points and the set $\mathscr{L}$ of lines. In particular, this shows that the point and line sets of a (topological) stable plane $\mathscr{P}$ admit at most one smooth structure such that $\mathscr{S}$ becomes a smooth stable plane. The investigation of central and axial collineations in the case of (topological) stable planes due to R. Löwen ([25], [26], [27]) is continued for smooth stable planes. Many results of [26] which are only proved for low dimensional planes $(\operatorname{dim} \mathscr{S} \leq 4)$ are transferred to smooth stable planes of arbitrary finite dimension. As an application of these transfers we show that the stabilizers $\Gamma_{[c, c]}^{1}$ and $\Gamma_{[A, A]}^{1}$ (see (3.2) Notation) are closed, simply connected, solvable subgroups of Aut $(\mathscr{S})$ (Corollary (4.17)). Moreover, we show that $\Gamma_{[c, c]}$ is even abelian (Theorem (4.18)). In the closing section we investigate the behaviour of reflections.
\end{abstract}

1991 Mathematics Subject Classification: 51H25, 51A40.

\section{Introduction}

In the early 30's Kolmogoroff was the first who combined projective spaces with topological structures. In the mid-fifties, Skornyakov (1954) and Freudenthal (1957) published two papers on topological planes, and it was Helmut Salzmann who started the systematic investigation of topological plane geometries in 1955 . Remembering the initial task of Euclid and Hilbert to characterize the euclidean plane $\mathscr{E}$, it is natural to consider not only the topology of $\mathscr{E}$ but also its differentiable structure. This leads from topological geometry to smooth geometry, i.e. incidence geometry on smooth manifolds such that the geometric operations are smooth.

In sharp contrast to the case of (compact) topological planes, only a few papers on smooth geometries have been published up to now. The pioneer of smooth geometry, S. Breitsprecher, wrote three papers in the late 60's, [7], [8], [9]. In 1972, 
Dieter Betten published a paper [2] on differentiable two-dimensional projective planes, in which he answered a question of Hellmuth Kneser by showing that the proper Moulton planes cannot be turned into smooth projective planes. After a period of 14 years where nothing was published about smooth geometry, Hermann Hähl revived this field with two papers, [18] and [14], (one of them is a joint paper with Theo Grundhöfer) about spherical fibrations which lead to smooth translation planes defined over division algebras. The main result of these two papers is that every smooth translation plane defined over some division algebra is isomorphic to one of the classical projective planes $\mathscr{P}$, i.e. the projective planes defined over the real, complex, quaternion, or octonion numbers, which we abbreviate by $\mathbb{R}, \mathbb{C}$, $H$, and $\mathbb{O}$, respectively. Joachim Otte generalized this result in 1992 to arbitrary translation planes, see [33] and [34].

We introduced the concept of a smooth stable plane in [4]. In this paper we will study collineations and collineation groups of smooth stable planes. We recall the most basic definitions of [4].

(1.1) Definition. A linear space is a triple $\mathscr{P}=(P, \mathscr{L}, \mathscr{F})$ of sets $P, \mathscr{L}$ and $\mathscr{F}$, where $P$ denotes the set of points, $\mathscr{L}$ is the set of lines and $\mathscr{F} \subseteq P \times \mathscr{L}$ is the set of flags, such that for every pair of distinct points $p, q$ there is exactly one joining line $L \in \mathscr{L}$, i.e. $(p, L),(q, L) \in \mathscr{F}$. If $(p, L) \in \mathscr{F}$, we will say that $p$ and $L$ are incident, or that $p$ lies on $L$, or that $L$ passes through $p$.

(1.2) Definition. A stable plane $\mathscr{S}$ is a linear space $(P, \mathscr{L}, \mathscr{F})$ which satisfies the following three axioms:

(S1) There are Hausdorff topologies on both $P$ and $\mathscr{L}$ that are neither discrete nor anti-discrete such that the join map $\vee$ and the intersection map $\wedge$ are continuous. Moreover, the domain $\mathcal{O}$ of the intersection map is an open subset of $\mathscr{L} \times \mathscr{L}$.

(S2) The topology on $P$ is locally compact and has positive finite covering dimension.

(S3) $\mathscr{S}$ contains a quadrangle.

A smooth stable plane $\mathscr{S}$ is a stable plane $(P, \mathscr{L}, \mathscr{F})$ such that $P$ and $\mathscr{L}$ are smooth manifolds and such that the join and intersection mappings are smooth on their (respective) domains.

We will use the words smooth and $\mathrm{C}^{\infty}$-differentiable synonymously. Note, however, that the results of [4] as well as of the present paper are still valid for $\mathrm{C}^{2}$-differentiable stable planes.

(1.3) Examples. Every subgeometry defined on some nonempty open subset of the point set of one of the classical projective planes (see the introduction for the definition of a classical plane) is a smooth stable plane. This includes the hyperbolic 
planes. Otte, [33], has shown that there is a plethora of non-classical smooth affine and projective planes. He describes these examples algebraically in terms of a coordinatizing ternary field. However, his examples are in general rather unaccessible from a geometric point of view. More explicit examples of smooth affine and stable planes will be given in forthcoming papers.

For the readers' convenience the following theorem summarizes some properties of stable planes. Proofs can be found in [25] and [28].

(1.4) Theorem. Let $\mathscr{S}=(P, \mathscr{L}, \mathscr{F})$ be a stable plane and let $p \in P, L \in \mathscr{L}$.

a) The point row $L$ is a closed subset of the set $P$ of points, the line pencil $\mathscr{L}_{p}$ is compact, and the flag space $\mathscr{F}$ is a closed subset of $P \times \mathscr{L}$.

b) The set $\mathscr{L}$ of lines and the line pencils $\mathscr{L}_{p}$ are connected.

c) $\operatorname{dim} L=\operatorname{dim} \mathscr{L}_{p}=l=2^{k}$ with $k \in\{0,1,2,3\}$, and $\operatorname{dim} P=\operatorname{dim} \mathscr{L}=2 l$, $\operatorname{dim} \mathscr{F}=3 l$, where $\operatorname{dim}$ denotes the covering dimension. The stable plane $\mathscr{S}$ is said to be 2 l-dimensional.

d) $\operatorname{dim} U=\operatorname{dim} X$ for every open subset $U$ of $X \in\left\{L, \mathscr{L}_{p}, P, \mathscr{L}, \mathscr{F}\right\}$.

Throughout this paper, let $\mathscr{S}=(P, \mathscr{L}, \mathscr{F})$ denote a smooth stable plane of dimen$\operatorname{sion} n=2 l$.

\section{Collineations}

The main topic of this chapter is the investigation of smooth collineations (i.e. automorphisms) of smooth stable planes. We show that every continuous collineation of such a plane is in fact a smooth collineation. This implies that the group $\Gamma$ of all continuous collineations is a Lie group with respect to the compact-open topology. In particular, the smooth structures of a smooth stable plane are uniquely determined by the underlying (topological) stable plane.

(2.1) Definition. Let $\mathscr{S}=(P, \mathscr{L}, \mathscr{F})$ and $\mathscr{S}^{\prime}=\left(P^{\prime}, \mathscr{L}^{\prime}, \mathscr{F}^{\prime}\right)$ be two smooth stable planes. A collineation $\gamma: \mathscr{S} \rightarrow \mathscr{S}^{\prime}$ is a pair $\left(\gamma_{P}, \gamma_{\mathscr{L}}\right)$ of bijections $\gamma_{P}: P \rightarrow P^{\prime}$ and $\gamma_{\mathscr{L}}: \mathscr{L} \rightarrow \mathscr{L}^{\prime}$ such that $\left(\gamma_{P} \times \gamma_{\mathscr{L}}\right)(\mathscr{F}) \subseteq \mathscr{F}^{\prime}$. We write $\gamma_{\mathscr{F}}$ instead of $\left(\gamma_{P} \times \gamma_{\mathscr{L}}\right)$. If $\mathscr{S}^{\prime}=\mathscr{S}$, then $\gamma$ is called a collineation of $\mathscr{S}$. A collineation $\gamma$ is called continuous (smooth), if the mappings $\gamma_{P}$ and $\gamma_{\mathscr{L}}$ are continuous (smooth). Most of the time, we will just use the symbol $\gamma$ instead of $\gamma_{P}, \gamma_{\mathscr{L}}$ or $\gamma_{\mathscr{F}}$.

Since the point rows of a stable plane $\mathscr{S}=(P, \mathscr{L}, \mathscr{F})$ are submanifolds of $P$ by [4], (2.6), we may apply the notion of smoothness to the restriction $\left.\gamma_{P}\right|_{L}$ of a collineation $\gamma: \mathscr{S} \rightarrow \mathscr{S}^{\prime}$ to some point row $L$.

(2.2) Proposition. Let $\gamma: \mathscr{S} \rightarrow \mathscr{S}^{\prime}$ be a collineation. If there exists a line $L \in \mathscr{L}$ such that the restriction $\left.\gamma_{P}\right|_{L}$ is smooth, then $\gamma$ is a smooth collineation. 
Proof. Let $L \in \mathscr{L}$ such that $\left.\gamma_{P}\right|_{L}$ is a smooth mapping.

1) We first show that $\left.\gamma_{P}\right|_{K}$ is smooth for every line $K \in \mathscr{L}$. Fix a line $K \in \mathscr{L} \backslash\{L\}$ and let $u$ be some point on $K$ not incident with $L$. Choose some point $v \in L$ and select another point $p \in(u \vee v) \backslash\{u, v\}$ which exists by (S3). Then, by axiom (S1), the projectivity

$$
\eta=\eta_{p, L}^{-1} \circ \eta_{p, K}: K \rightarrow L: x \mapsto(x \vee p) \wedge L
$$

is defined on some open neighborhood $U$ of $u$ in $K$. Moreover, by [4], (2.7), the map $\eta: U \rightarrow \eta(U)$ is a diffeomorphism and $\eta(U)$ is an open subset of $L$. Since $\gamma$ is a collineation, we have the commuting diagram

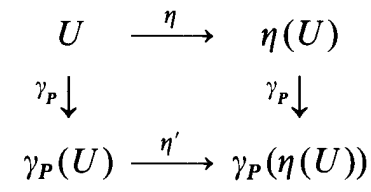

where $\eta^{\prime}$ is the projectivity of $\mathscr{S}^{\prime}$ which corresponds to $\eta$, i.e. we have

$$
\eta^{\prime}: \gamma_{P}(K) \rightarrow \gamma_{P}(L): x \mapsto\left(x \vee \gamma_{P}(p)\right) \wedge \gamma_{\mathscr{L}}(L)
$$

Hence, the restriction $\left.\gamma_{P}\right|_{U}: U \mapsto \gamma_{P}(U)$ is smooth and thus $\left.\gamma_{P}\right|_{K}$ is smooth as well.

2) According to [4], (2.8) and (2.9), a collineation $\gamma$ is smooth if and only if one of the maps $\gamma_{P}$ and $\gamma_{\mathscr{L}}$ is smooth. Hence, in order to prove the proposition, we only have to check that $\gamma_{\mathscr{L}}$ is smooth. Let $M \in \mathscr{L}$. Choose two distinct points $p, q$ on $M$, and let $K \in \mathscr{L}_{p} \backslash\{M\}$ and $L \in \mathscr{L}_{q} \backslash\{M\}$. By [4], (2.9) there is an open neighborhood $\mathscr{U}_{M}$ of $M$ in $\mathscr{L}$ such that $v_{K, L}: \mathscr{U}_{M} \rightarrow K \times L: X \mapsto(K \wedge X, L \wedge X)$ is a diffeomorphism onto some open subset of $K \times L$. Setting $a^{\prime}:=\gamma(a)$ for $a \in P \cup \mathscr{L}$, we have the diagram

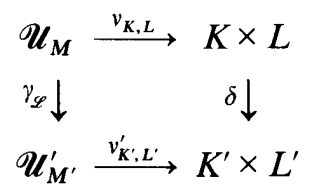

where $v_{K^{\prime}, L^{\prime}}^{\prime}: \mathscr{U}_{M^{\prime}}^{\prime} \rightarrow K^{\prime} \times L^{\prime}$ is the diffeomorphism given by [4], (2.9) with respect to $\mathscr{S}^{\prime}$ and $\delta=\left.\gamma_{P}\right|_{K} \times\left.\gamma_{P}\right|_{L}$. This diagram commutes, because for $N \in \mathscr{U}_{M}$ we have

$$
\begin{aligned}
\left(\left.\gamma_{P}\right|_{K} \times\left.\gamma_{P}\right|_{L}\right) \circ v_{K, L}(N) & =\left(\gamma_{P}(K \wedge N), \gamma_{P}(L \wedge N)\right) \\
& =\left(\gamma_{\mathscr{L}}(K) \wedge \gamma_{\mathscr{L}}(N), \gamma_{\mathscr{L}}(L) \wedge \gamma_{\mathscr{L}}(N)\right) \\
& =\left(K^{\prime} \wedge N^{\prime}, L^{\prime} \wedge N^{\prime}\right) \\
& =v_{K^{\prime}, L^{\prime}}^{\prime} \circ \gamma_{\mathscr{L}}(N) .
\end{aligned}
$$


By what we have proved in 1), this implies that $\gamma_{\mathscr{L}}$ is smooth at $M$. Since $M \in \mathscr{L}$ can be chosen arbitrarily, the claim follows.

The following main theorem shows that there is, in fact, no difference between continuous collineations and smooth collineations.

(2.3) Theorem. Every continuous collineation $\gamma: \mathscr{S} \rightarrow \mathscr{S}^{\prime}$ between two smooth stable planes is a smooth collineation.

Proof. By Proposition (2.2) we have to check that the restriction $\left.\gamma_{P}\right|_{L}$ is smooth for some line $L \in \mathscr{L}$. Fix a point $o$ incident with $L$. By [25], (1.21), there is a quadrangle $\diamond=\{o, u, v, e\}$ in $P$ with $u \in L$, and there is an open neighborhood $U$ of $o$ in $L$ such that a ternary operation $\tau: U \times U \times U \rightarrow L$ is defined in terms of join and intersection. Thus $\tau$ is smooth. Setting $x+y:=\tau(1, x, y)$ for $x, y \in U$, where $1=(v \vee e) \wedge L$, we get a smooth local $\mathrm{H}$-space $\mathscr{H}=(L, U, o,+)$ in the sense of [5], (1.2). Since the image $\gamma(\mathscr{S})=\mathscr{S}^{\prime}$ is a stable plane, the quadrangle $\diamond$ is mapped by $\gamma_{P}$ onto a quadrangle $\diamond^{\prime}=\left\{o^{\prime}, u^{\prime}, v^{\prime}, e^{\prime}\right\} \subseteq P^{\prime}$. As before, we obtain a smooth local H-space $\mathscr{H}^{\prime}=\left(L^{\prime}, U^{\prime}, o^{\prime},+^{\prime}\right)$ on the point row $L^{\prime}=\gamma_{P}(L)$. Since $\gamma$ is a collineation and since the operations + and $+^{\prime}$ are defined in purely geometrical terms, the map $\left.\gamma_{P}\right|_{L}$ is a continuous homomorphism between the smooth local H-spaces $\mathscr{H}$ and $\mathscr{H}^{\prime}$. By [5], (1.8), we conclude that $\left.\gamma_{P}\right|_{L}$ is smooth on some neighborhood of $o$. By varying the point $o$ on $L$, this shows that the map $\left.\gamma_{P}\right|_{L}$ is smooth at every point of $L$. This completes the proof.

Let $\Gamma$ be the group of all continuous collineations of the smooth stable plane $\mathscr{S}$. We will refer to $\Gamma$ as the automorphism group or the collineation group of $\mathscr{P}$. The compact-open topologies of $\Gamma$ on $P$, on $\mathscr{L}$, and on $\mathscr{F}$ coincide. In this topology, the group $\Gamma$ is locally compact, and $\Gamma$ acts as a topological transformation group on $P$ as well as on $\mathscr{L}$ by [25], (2.9). According to Theorem (2.3), the group $\Gamma$ consists of diffeomorphisms of $P$ and of $\mathscr{L}$. Since $\mathscr{L}$ is connected (see Theorem (1.4)), we get the following result from [31], Chap. V, Th. 2.

(2.4) Corollary. The automorphism group $\Gamma$ of a smooth stable plane $\mathscr{S}=(P, \mathscr{L}, \mathscr{F})$ is a smooth Lie transformation group on the manifold $P$ of points, the manifold $\mathscr{L}$ of lines, and the manifold $\mathscr{F}$ of flags with respect to the compact-open topology.

As another immediate consequence we have

(2.5) Corollary. A (topological) stable plane $\mathscr{S}$ admits at most one smooth structure on the set $P$ of points and on the set $\mathscr{L}$ of lines, respectively, such that $\mathscr{S}$ becomes a smooth stable plane.

Remark. The proofs of Proposition (2.2) and Theorem (2.3) are still valid for injective continuous homomorphisms $\gamma=\left(\gamma_{P}, \gamma_{\mathscr{L}}\right): \mathscr{S} \rightarrow \mathscr{S}^{\prime}$, i.e. $\gamma_{P}$ and $\gamma_{\mathscr{L}}$ are injective con- 
tinuous maps such that $\left(\gamma_{P} \times \gamma_{\mathscr{L}}\right)(\mathscr{F}) \subseteq \mathscr{F}^{\prime}$ : the arguments used in the proof of Proposition (2.2) do not use the fact that $\gamma$ is a surjection. In order to prove Theorem (2.3) for injective continuous homomorphisms we only need to check that the image $\gamma(\diamond)$ of a quadrangle $\diamond$ is again a quadrangle. But this is true since $\gamma_{\mathscr{L}}$ is injective and thus has to map the four distinct vertices of $\diamond$ to four distinct vertices of $\gamma(\diamond)$. Since we are mainly interested in the automorphism group $\Gamma$ of a smooth stable plane, we restrict our attention to isomorphisms.

\section{Basic results on central collineations}

Central and axial collineations of topological stable planes behave quite differently compared to their relatives in projective planes. For example, a central collineation of a stable plane need not have an axis, because you can remove the points of an existing axis and get a new stable plane by restriction. However, in the case of smooth stable planes differentiability does not guarantee any fixed elements, but makes certain proofs work just as if these fixed elements existed.

(3.1) Definition. Let $\mathscr{S}=(P, \mathscr{L}, \mathscr{F})$ be a smooth stable plane. A collineation $\gamma$ of $\mathscr{S}$ is called a central collineation with center $p$ if $\gamma$ fixes every line through $p$. Dually, a collineation $\gamma$ is called axial with axis $L$ if every point on $L$ is a fixed point of $\gamma$.

Note that it is not necessary to require a central or axial collineation to be continuous or even smooth. In fact, any such collineation is continuous by Löwen [25], (3.2), and thus is smooth by Theorem (2.3). In contrast to the projective case, a central collineation of a stable plane need not have an axis and an axial collineation may have no center. Moreover, a central collineation $\gamma$ may fix every point of an open subset of some line $A$ rather than every point of $A$. Such a central collineation is called semi-axial and $A$ is referred to as its semi-axis, see Löwen [26], §3.

(3.2) Notation. For any point $c \in P$ we denote by $\Gamma_{[c]}$ the subgroup of $\Gamma$ consisting of all central collineations $\gamma \in \Gamma$ with center $c$. The group $\Gamma_{[A]}$ for some line $A$ is defined dually. Moreover, we use the abbreviations $\Gamma_{[c, A]}:=\Gamma_{[c]} \cap \Gamma_{[A]}$ and $\Gamma_{[c, c]}:=\left\langle\bigcup_{A \in \mathscr{L}_{c}} \Gamma_{[c, A]}\right\rangle$. The group $\Gamma_{[A, A]}$ is defined dually. If $(c, A)$ is a flag, then the elements of $\Gamma_{[c, A]}$ are called elations, otherwise they are called homologies. Note that unlike in the projective case, where every central (axial) collineation has an axis (a center), it is not clear whether or not the generating sets $\bigcup_{A \in \mathscr{L}_{c}} \Gamma_{[c, A]}$ and $\bigcup_{c \in A} \Gamma_{[c, A]}$ form subgroups of $\Gamma$. Nevertheless, as we will see later, this turns out to be true for the groups $\Gamma_{[c, c]}$ (see Corollary (4.9)).

We turn to local versions of the aforementioned groups. Let $(c, A)$ be a flag. We define $\Gamma_{[c, A]}^{s}$ as the set of all central collineations with center $c$ and semi-axis $A$ that fix every point of some open neighborhood $O$ of $p$ in $A$. We refer to the elements of $\Gamma_{[c, A]}^{s}$ as semi-elations. Clearly, the set $\Gamma_{[c, A]}^{s}$ of semi-elations forms a group. As in the case of elations we put $\Gamma_{[c, c]}^{s}:=\left\langle\bigcup_{A \in \mathscr{L}_{c}} \Gamma_{[c, A]}^{s}\right\rangle$ and $\Gamma_{[A, A]}^{s}:=\left\langle\bigcup_{c \in A} \Gamma_{[c, A]}^{s}\right\rangle$. If, on the other hand, $(c, A)$ is an anti-flag (i.e. not a flag), we define $\Gamma_{[c, A]}^{s}$ to be the 
set of all axial collineations with axis $A$ that fix every line $c \vee x$ for $x \in A$. We call such collineations semi-homologies and we speak of $c$ as their semi-center. We will show, however, that every semi-homology (semi-elation) of a smooth stable plane is in fact a homology (an elation); see Theorem (3.5) and Corollary (4.6).

(3.3) Theorem. For every point $p \in P$ and for every line $L \in \mathscr{L}$ the derivation mappings $\mathrm{D}_{p}: \Gamma_{p} \rightarrow \operatorname{GL}\left(\mathrm{T}_{p} P\right): \gamma \mapsto \gamma_{P}^{\prime}(p)$ and $\mathrm{D}_{L}: \Gamma_{L} \rightarrow \mathrm{GL}\left(\mathrm{T}_{L} \mathscr{L}\right): \gamma \mapsto \gamma_{\mathscr{L}}^{\prime}(L)$ are Lie group homomorphisms.

Proof. The stabilizers $\Gamma_{p}$ and $\Gamma_{L}$ are closed subgroups of $\Gamma$. Since the latter group is a Lie group by Corollary (2.4), these stabilizers are Lie groups as well. Because any continuous homomorphism between Lie groups is a Lie group homomorphism (i.e. it is analytic), it suffices to prove that $D_{p}$ and $D_{L}$ are continuous mappings. The set $P$ of points is locally connected by Löwen [25], (1.4) and (1.11), and thus every connected component of $P$ is an open subset of $P$. Hence the connected component $P_{p}$ of $p$ in $P$ becomes a smooth stable plane via restriction. Moreover, the stabilizer $\Gamma_{p}$ leaves $P_{p}$ invariant and acts effectively on $P_{p}$. Applying [31], Theorem, p. $208 \mathrm{ff}$ to the transformation group $\left(\Gamma_{p}, P_{p}\right)$, we conclude that the homomorphism $\mathrm{D}_{p}: \Gamma_{p} \rightarrow \mathrm{GL}\left(\mathrm{T}_{p} P\right): \gamma \mapsto \gamma_{p}^{\prime}(p)$ is continuous. Since the set $\mathscr{L}$ of lines is (arcwise) connected, the continuity of $\mathrm{D}_{L}$ follows immediately. In order to show that the line space $\mathscr{L}$ is arcwise connected, we use the fact that every line is locally arcwise connected (Löwen [25], (1.12)). Hence every line pencil $\mathscr{L}_{p}$ is locally arcwise connected as well ([25], (1.7)) and thus $\mathscr{L}_{p}$ is arcwise connected, since it is connected ([25], (1.14)). Now choose two lines $K \neq L$ and fix two distinct points $k \in K$ and $l \in L$. Then there are paths from $K$ to $k \vee l$ and from $k \vee l$ to $L$. Concatenating these two paths gives a path from $K$ to $L$ in $\mathscr{L}$. This proves the arcwise connectedness of $\mathscr{L}$ as well as the theorem.

When passing from (topological) stable planes to smooth stable planes $\mathscr{P}=(P, \mathscr{L})$, one of the most useful additional structures you get are the topological translation planes $\mathscr{A}_{p}$ defined on the tangent spaces $\mathrm{T}_{p} P$ at any point $p \in P$. These planes are defined as follows. The set of points is just $\mathrm{T}_{p} P$ and the collection $\mathscr{P}_{p}:=\left\{\mathrm{T}_{p} L \mid L \in \mathscr{L}_{p}\right\}$ of $l$-dimensional subspaces serves as the line pencil at the origin $0 \in \mathrm{T}_{p} P$. By [4], we have (SP1) $\bigcup_{K \in \mathscr{L}_{p}} \mathrm{~T}_{p} K=\mathrm{T}_{p} P$ and (SP2) $\mathrm{T}_{p} L \oplus \mathrm{T}_{p} K=\mathrm{T}_{p} P$ for any two distinct lines $K, L \in \mathscr{L}_{p}$. Any family of $l$-dimensional subspaces of a $2 l$-dimensional vector space $V$ that satisfies these two axioms is called a spread on $V$. It is a well-known result that every spread $\mathscr{S}$ on $V$ defines a translation plane $\mathscr{A}$ on the point set $V$ by putting $\mathscr{L}:=\{W+v \mid W \in \mathscr{S}, v \in V\}$ as the set of lines of $\mathscr{A}$. By [4], (3.5), the planes $\mathscr{A}_{p}$ are even locally compact affine translation planes. We will refer to $\mathscr{A}_{p}$ as the tangent translation plane at $p$ and to $\mathscr{S}_{p}$ as the tangent spread at $p$. The projective closure $\mathscr{P}_{p}$ of $\mathscr{A}_{p}$ is a compact connected projective translation plane and we denote by $L_{\infty}$ the line at infinity of $\mathscr{P}_{p}$ with respect to the affine plane $\mathscr{A}_{p}$. Note that the dually defined tangent incidence structure $\mathscr{A}_{L}$ at some line $L$ need not be an affine plane. While axiom (SP2) is still valid, axiom (SP1) may not be true 
anymore. Nevertheless, the incidence structure $\mathscr{A}_{L}$ is the dual of a so-called shear plane which is a special kind of a stable plane. In fact, shear planes can be regarded as a generalization of translation planes in the category of stable planes, see [4], section 4 , and [24].

If $\Gamma$ fixes some point $p$, then the derivation mapping $\mathrm{D}_{p}: \Gamma \rightarrow \mathrm{GL}\left(\mathrm{T}_{p} P\right)$ maps $\Gamma$ to Aut $\left(\mathscr{A}_{p}\right)$. In this way, every collineation $\gamma \in \Gamma_{p}$ induces an action on the tangent spread $\mathscr{S}_{p}$. This action is given by $\mathrm{D}_{p} \gamma\left(\mathrm{T}_{p} L\right)=\mathrm{T}_{p}\left(L^{\gamma}\right)$ for every line $L \in \mathscr{L}_{p}$. Thus, the transformation groups $\left(\Gamma_{p}, \mathscr{L}_{p}\right)$ and $\left(\mathrm{D}_{p}\left(\Gamma_{p}\right), \mathscr{S}_{p}\right)$ are equivalent via the equivariant homeomorphism $\mathscr{L}_{p} \rightarrow \mathscr{S}_{p}: L \mapsto \mathrm{T}_{p} L$. Since $\mathrm{D}_{p} \gamma$ is a linear map on $\mathrm{T}_{p} P$ which acts on the tangent spread $\mathscr{S}_{p}$, the group $\mathrm{D}_{p} \Gamma_{p}$ consists of automorphisms of $\mathscr{P}_{p}$ which fix the origin 0 of $\mathrm{T}_{p} P$ and the line $L_{\infty}$ at infinity.

In this and the following chapter we want to determine the kernel of $D_{p}$ (see Theorem 4.13) and then utilize the map $D_{p}$ in order to derive some results on the structure of elation groups and of reflections.

(3.4) Lemma. If $\gamma \in \Gamma$ fixes three lines $L_{1}, L_{2}, L_{3} \in \mathscr{L}_{p}$ for some point $p \in P$, then the set $F_{\gamma}$ of fixed lines of $\gamma$ acting on $\mathscr{L}_{p}$ is a homology $m$-sphere $S$ with $m \geq 1$.

Proof. By Theorem (1.4), the line pencil $\mathscr{L}_{p}$ is homeomorphic to $\mathbb{S}_{l}$. Assume that $\gamma$ acts non-trivially on $\mathscr{L}_{p}$. The image $\mathrm{D}_{p} \gamma$ fixes the three different lines $\mathrm{T}_{p} L_{i}$ of the tangent translation plane $\mathscr{A}_{p}$. By Hähl [16], (2.1), the group of automorphisms of $\mathscr{A}_{p}$ that fix the three lines $\mathrm{T}_{p} L_{i}$ induces a non-trivial compact Lie group $\Phi$ on the tangent spread $\mathscr{P}_{p}$. Thus the group $\Psi:=\overline{\left\langle\mathrm{D}_{p} \gamma\right\rangle}$ contains some torus subgroup $\mathrm{T}$ of $\Phi$ or $\mathrm{D}_{p} \gamma$ has finite order $r$. Suppose that $\Psi$ contains a torus group T. Then we have $\left|F_{\top}\right| \geq 3$ since $\left|F_{\gamma}\right| \geq 3$. Thus, by Floyd [13], (4.1) and [12], (5.2), the fixed point set $F_{\mathrm{T}}$ of a torus group T acting on a (homology) sphere with fixed points is a homology $m$-sphere with $m \geq 1$. Since $\mathrm{D}_{p} \gamma$ topologically generates $\Psi$, we infer that $F_{\gamma} \approx F\left(\mathrm{D}_{p} \gamma\right):=\left\{S \in \mathscr{S}_{p} \mid S^{\mathrm{D}_{p} \gamma}=S\right\}=F_{\psi}$. In particular, we have $F_{\psi}=F_{\mathrm{T}}$, whence $F_{\gamma}$ is a homology $m$-sphere. If, on the other hand, the order $r$ of $\mathrm{D}_{p} \gamma$ is finite, we let $r=p_{1}^{s_{1}} \cdots \cdots \cdot p_{k}^{s_{k}}$ be the decomposition of $r$ into its prime powers, and we put $r_{i}:=p_{1}^{s_{1}} \cdots \cdots p_{i}^{s_{i}}$ as well as $\varphi_{i}:=\left(\mathrm{D}_{p} \gamma\right)^{r_{i}}$. By Liao [23], the fixed point set $F_{k-1}$ of $\varphi_{k-1}$ is a homology sphere of positive dimension (remember that $\left|F_{\gamma}\right| \geq 3$ by hypothesis). Because $\varphi_{k-i-1}$ acts on $F_{k-1}$, we inductively conclude that $F\left(\mathrm{D}_{p} \gamma\right)$ is a homology $m$-sphere with $m \geq 1$. Hence the claim of the lemma follows.

The next theorem is in the spirit of R. Baer's characterization of quasi-perspectivities of projective planes (Baer, [1]); see Stroppel, [38], Theorem 5 for an extension of Baer's theorem to stable planes.

(3.5) Theorem. If $\gamma \in \Gamma$ fixes an open subset of some line pencil $\mathscr{L}_{c}$, then $\gamma$ is a central collineation with center $c$. In particular, every semi-homology is in fact a homology.

Proof. By Lemma (3.4), the set $F_{\gamma}$ of fixed lines of $\gamma$ acting on $\mathscr{L}_{c}$ is a homology $m$-sphere $S$. Since $\gamma$ fixes an open subset $U$ of $\mathscr{L}_{c}$, we have $m=\operatorname{dim} U=\operatorname{dim} \mathscr{L}_{c}=l$ 
according to Theorem (1.4). We will show that $S=\mathscr{L}_{c}$. The following part of the proof is due to Linus Kramer. Let $L$ be some interior line of $S$ and choose some neighborhood $U \subset S$ around $L$. Denote by $\imath: S \hookrightarrow \mathscr{L}_{c}$ the inclusion map and consider the diagram of singular homology groups over $\mathbb{Z}_{2}$

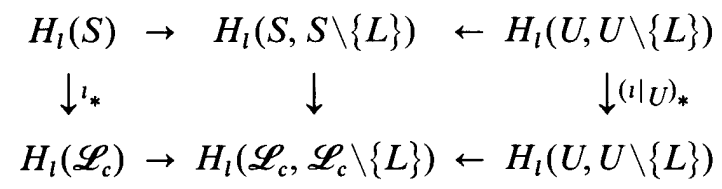

By invariance of domain, the restriction $\left.{ }^{\imath}\right|_{U}$ is a homeomorphism and thus the induced map $\left(\left.l\right|_{U}\right)_{*}$ is an isomorphism. Since $S$ and $\mathscr{L}_{c}$ are $\mathbb{Z}_{2}$-orientable, the horizontal arrows on the left hand side are isomorphisms, while the horizontal arrows on the right hand side represent excision isomorphisms. In particular, the induced map $l_{*}$ is an isomorphism, too. Suppose that $l$ is not surjective. Choose some line $K \in \mathscr{L}_{c} \backslash S$. Then $\imath$ factors as $\imath: S \rightarrow \mathscr{L}_{c} \backslash\{K\} \rightarrow \mathscr{L}_{c}$ in contradiction to $H_{l}\left(\mathscr{L}_{c} \backslash\{K\}\right) \cong H_{l}\left(\mathbb{R}^{l}\right)=\{0\}$. Thus we have proved that $F_{\gamma}=S=\mathscr{L}_{c}$. Now, the second statement follows from [4], (1.7).

(3.6) Theorem. For every point $p \in P$ the kernel of the mapping $\mathrm{D}_{p}$ consists of central collineations whose semi-axes, if they exist, are incident with $p$. Dually, for every line $L \in \mathscr{L}$ the kernel of the mapping $\mathrm{D}_{L}$ consists of axial collineations whose centers, if they exist, are lying on $L$.

Proof. Let $p \in P$ and $\gamma \in \Gamma_{p}$. If $\gamma$ acts non-trivially on the line pencil $\mathscr{L}_{p}$, then $\mathrm{D}_{p} \gamma$ acts non-trivially on the tangential spread $\mathscr{S}_{p}$ and hence $\gamma \notin \operatorname{ker} \mathrm{D}_{p}$. Thus we may assume that $\gamma$ is a central collineation (with center $p$ ). Moreover, suppose that $\gamma$ has a semi-axis $A$ not incident with $p$. Then $A$ is an axis of $\gamma$, since $\gamma$ fixes every line through $p$. We have to verify that $\gamma$ is not contained in the kernel of $\mathrm{D}_{p}$. Indeed, fix two distinct points $u, v$ on $A$, and put $K_{u}:=p \vee u$ and $K_{v}:=p \vee v$. The triangle $\{p, u, v\}$ defines a smooth local loop $T$ in the sense of [3] on a neighborhood $V \subseteq K_{u}$ of $p$, see Löwen [25], (1.21). Since $\gamma$ fixes its axis $A$ pointwise, the restriction of $\gamma$ to $U$ is a homomorphism of $T$. We now construct a coordinate system $(h, U)$ of $P$ around $p$ such that $\left.\gamma\right|_{U \cap K_{u}}$ is a linear map, which shows that $\mathrm{D}_{p} \gamma \neq \mathbb{1}$. In order to define $h$, we use two charts $h_{K_{u}}$ and $h_{K_{v}}$ of the lines $K_{u}$ and $K_{v}$ around $p$. Applying [4], (1.11) to these charts we get a chart $h$ of $P$ around $p$ satisfying $\left.h\right|_{K_{u}}=h_{K_{u}}$. By the results of Kozma, [21] and [22], there is a so-called canonical coordinate system $h_{K_{u}}$ for the local loop $T$, and with respect to this chart the restriction $\left.\gamma\right|_{U \cap K_{u}}$ is locally a linear map. Taking an arbitrary chart $h_{K_{v}}$ on $K_{v}$ around $p$, this proves the first part of the theorem.

For the dual assertion, we assume that $\gamma \in \operatorname{ker} D_{L}$ is an axial collineation with axis $L$ and semi-center $p \notin L$. By Theorem (3.5), the point $p$ is in fact a center of $\gamma$. The map $\vee_{u}: V \rightarrow \mathscr{V} \subseteq \mathscr{L}_{u}: x \mapsto x \vee u$ is a diffeomorphism between $V$ and an open neighborhood $\mathscr{V}$ of $p \vee u$ (see [4], (1.7)) that transfers the smooth local loop 
structure $T$ on $V$ to a smooth local loop structure $\tilde{T}$ on $\mathscr{V}$. Clearly, the collineation $\gamma$ is a homomorphism of $T$ again. Since $\gamma$ commutes with $\vee_{u}$, the map $\gamma$ induces a homomorphism of $\tilde{T}$ and we may proceed as before.

(3.7) Proposition. Let $(p, L)$ be a flag. Then $\Gamma_{[p, L]}^{s} \leq \operatorname{ker} \mathrm{D}_{p}$ and $\Gamma_{[p, L]} \leq \operatorname{ker} \mathrm{D}_{L}$.

Proof. Let $\gamma \in \Gamma_{[p, L]}^{s}$ for some flag $(p, L)$. Since $\gamma$ fixes every line of $\mathscr{L}_{p}$, the derivative $\mathrm{D}_{p} \gamma$ fixes every element of the tangential spread $\mathscr{P}_{p}$. According to Hähl [18], (2.1) and (2.3), we may select two spread elements $V, W \in \mathscr{S}_{p}$ and a basis $\mathscr{B}:=\left\{v_{1}, \ldots, v_{2 l}\right\}$ of $\mathrm{T}_{p} P$ such that $\mathrm{T}_{p} L=\left\langle v_{1}, \ldots, v_{l}\right\rangle, V=\left\langle v_{l+1}, \ldots, v_{2 l}\right\rangle$, and $W=\left\langle v_{1}+v_{l+1}, \ldots, v_{l}+v_{2 l}\right\rangle$. Since $\mathrm{D}_{p} \gamma$ leaves the subspaces $\mathrm{T}_{p} P, V$, and $W$ invariant, the matrix representation of $\mathrm{D}_{p} \gamma$ with respect to $\mathscr{B}$ is just $\left(\begin{array}{ll}A & 0 \\ 0 & A\end{array}\right)$, where $A \in \mathrm{GL}_{l} \mathbb{R}$. Because $\gamma$ is the identity on some neighborhood of $p$ in $L$, its derivative $\mathrm{D}_{p} \gamma$ is the identity on $\mathrm{T}_{p} L$, i.e., we have $A=1$. We omit the verification of the dual statement.

(3.8) Corollary. We have $\Gamma_{[p, p]}^{s} \subseteq \operatorname{ker} \mathrm{D}_{p}$ and $\Gamma_{[L, L]} \subseteq \operatorname{ker} \mathrm{D}_{L}$ for every point $p \in P$ and every line $L \in \mathscr{L}$.

Since every central collineation of a projective plane has an axis and vice versa, the last two results enable us to characterize the kernels of $D_{p}$ and $D_{L}$ for smooth projective planes in purely geometrical terms.

(3.9) Corollary. If $\mathscr{S}$ is a smooth projective plane, the kernel of $\mathrm{D}_{p}$ (of $\mathrm{D}_{L}$ ) consists exactly of all elations with center $p$ (with axis $L$ ).

We will generalize the above result to smooth stable planes in the next section. If a central or an axial collineation $\gamma \neq \mathbb{1}$ of a stable plane $\mathscr{S}$ fixes some triangle $\nabla:=\{o, u, v\}$, then $\gamma$ has both a center and an axis. Moreover, the center $o$ of $\gamma$ must be contained in $\nabla$ and the axis must be the line of this triangle opposite to the center, cf. Löwen [25], (3.4). Thus, by Theorem (3.6), the map $\mathrm{D}_{o}$ is a faithful continuous representation of $\Gamma_{\nabla}$.

(3.10) Corollary. The stabilizer $\Gamma_{\nabla}$ of a triangle $\nabla$ in a smooth stable plane $\mathscr{S}$ is a linear Lie group.

In the case of smooth projective planes, Theorem (2.3) together with Theorem (2.10) of [3] implies the compactness of the stabilizer of a quadrangle (recall that every quadrangle of a projective plane determines a ternary field, and that the stabilizer of a quadrangle can be regarded as the automorphism group of the associated ternary field). 
(3.11) Corollary. The stabilizer $\Gamma_{\diamond}$ of a quadrangle $\diamond$ in a smooth projective plane $\mathscr{S}$ is a compact Lie group.

(3.12) Corollary. Let $\Sigma=\Gamma_{(p, L)}$. If $(p, L)$ is a flag, then $\Sigma_{q}$ is a linear Lie group for every point $q \in P \backslash L$. If $(p, L)$ is not a flag, then $\Sigma$ itself is a linear Lie group.

Another corollary which can easily be derived from Theorem (3.6) deals with the structure of homology groups of stable planes (whereas the structure of elation groups is the subject of the next section). Beforehand we need a lemma.

(3.13) Lemma. Let $\wedge$ be a Lie group whose maximal torus subgroups are at most onedimensional. Then every one-parameter subgroup of $\wedge$ is closed in $\wedge$. Consequently, every connected subgroup of $\Lambda$ is closed.

Proof. Let $\mathbf{P} \leq \wedge$ be a one-parameter subgroup. Suppose that $\mathbf{P}$ is not closed. Then, by Hochschild [20], Chap. XVI, Prop. 2.3, the closure $\mathbf{P}$ of $P$ is compact. Since $\bar{P}$ is abelian, we conclude that $\bar{P}$ is contained in a torus subgroup $T$ of $\wedge$. Thus we have $\operatorname{dim} \bar{P}=1$ by hypothesis. Arcwise connectedness of $P$ and $\bar{P}$ now implies that $\bar{P}=P$, and hence $P$ is a closed subgroup of $\wedge$ after all. The last statement follows from [20], Chap. XVI, Theorem 2.4.

(3.14) Corollary. If $p \notin L$, then the connected component $\Gamma_{[p, L]}^{1}$ of the homology group $\Gamma_{[p, L]}$ can be embedded as a closed subgroup into the homology group of the classical projective plane of the respective dimension.

Proof. By Theorems (3.3) and (3.6), the derivation map $\mathrm{D}_{p}: \Gamma_{[p, L]}^{1} \rightarrow \operatorname{Aut}\left(\mathscr{P}_{p}\right)$ is a continuous injection, and since $\Gamma_{[p, L]}^{1}$ fixes every line through $p$, its image under $\mathrm{D}_{p}$ fixes every element of the tangent spread $\mathscr{S}_{p}$. The group $\mathrm{D}_{p} \Gamma_{[p, L]}$ cannot contain an elation, because any element of this group fixes every line through $o$ as well as the line $L_{\infty}$. Thus $\mathrm{D}_{p} \Gamma_{[p, L]}$ is a group of homologies of the tangent translation plane $\mathscr{P}_{p}$ with the origin $O$ as its center and the line $L_{\infty}$ at infinity as its axis. By Hähl [15], (3.2), the group Aut $\left(\mathscr{P}_{p}\right)_{\left[o, L_{\infty}\right]}$ is isomorphic to the multiplicative group of the kernel of the quasifield $Q$ which is associated to the spread $\mathscr{P}_{p}$. Since the kernel of

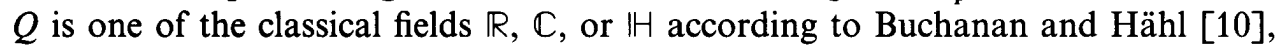
we can apply Lemma (3.13) in order to conclude that the restriction $\left.D_{p}\right|_{[p, L]} ^{1}$ is a closed mapping.

Recall that in the topological case it is not known whether the preceding corollary is true or not. Even for compact projective planes only a weaker result is known, see Hähl [17], (3.5). For stable planes of dimension at most 4, however, there is a proof of this corollary which is due to H.-P. Seidel [36]. 


\section{The structure of elation groups}

In this section we will elucidate the structure of the semi-elation groups $\Gamma_{[A, A]}^{s}$ and $\Gamma_{[c, c]}^{s}$. We will show that the connected components of these groups are simply connected solvable closed subgroups of the automorphism group $\Gamma$ of the smooth stable plane $\mathscr{S}$. Moreover, it will turn out that $\Gamma_{[c, c]}^{s}$ is even abelian. For the classical projective planes $P_{2} \mathbb{K}(\mathbb{K} \in\{\mathbb{R}, \mathbb{C}, \mathbb{H}, \mathbb{D}\}$ ) and, more generally, for affine or projective translation planes, these groups are isomorphic to vector groups. However, no proof of this claim is known even for compact connected projective planes. The most sophisticated results on the structure of (semi-)elation groups in the projective case are due to Hähl, [17], (3.7), and to M. Lüneburg, [29], VII, Satz 7.

For the investigation of the structure of semi-elation groups, we start with a lemma which is well-known for affine and projective planes.

(4.1) Lemma. Let $\mathscr{S}=(P, \mathscr{L})$ be a (topological) stable plane and let $c \in P$ and $A \in \mathscr{L}$. If $\Gamma_{[c, c]}^{s}$ contains elations with different axes, then $\Gamma_{[c, c]}^{s}$ is commutative. The dual statement holds for the group $\Gamma_{[A, A]}^{s}$.

Proof. It suffices to show that any two elements of the generating set $\bigcup_{A \in \mathscr{L}_{c}} \Gamma_{[c, A]}^{s}$ commute. Let $\gamma \in \Gamma_{[c, A]}^{s}$ and $\gamma^{\prime} \in \Gamma_{\left[c, A^{\prime}\right]}^{s}$ with $A \neq A^{\prime}$. Then we have $\gamma^{\prime-1} \gamma \gamma^{\prime} \in \Gamma_{[c, A]}^{s}$ and thus the commutator $\left[\gamma^{\prime}, \gamma\right]$ of $\gamma$ and $\gamma^{\prime}$ lies in $\Gamma_{[\mathfrak{c}, A]}^{s}$. With the same type of argument it is easily checked that $\left[\gamma^{\prime}, \gamma\right] \in \Gamma_{\left[c, A^{\prime}\right]}^{s}$. Since $\Gamma_{[c, A]}^{s} \cap \Gamma_{\left[c, A^{\prime}\right]}^{s}=[1]$ by Löwen [25], (3.4), we conclude that $\gamma$ commutes with $\gamma^{\prime}$. If, on the other hand, we have $\gamma, \gamma^{\prime \prime} \in \Gamma_{[c, A]}^{s}$, we choose $\gamma^{\prime} \in \Gamma_{\left[c, A^{\prime}\right]}^{s}$ with $A \neq A^{\prime}$. Since $\gamma \gamma^{\prime} \notin \Gamma_{[c, A]}^{s}$, we already know that $\gamma \gamma^{\prime}$ commutes with $\gamma^{\prime \prime}$. By associativity, we finally conclude that $\gamma$ and $\gamma^{\prime \prime}$ commute. Since the dual statement of [25], (3.4) is trivially true, the second part of the lemma is proved similarly.

It is not obvious that $\Gamma_{[c, A]}^{s}$ is a closed subgroup of $\Gamma$, since at first sight it seems to be possible that there is a sequence $\left(\gamma_{n}\right)_{n \in \mathbb{N}}$ in $\Gamma_{[c, A]}^{s}$ such that $\bigcap_{n \in \mathbb{N}} O_{n}=\{c\}$, where $O_{n}=\left\{p \in A \mid p^{y_{n}}=p\right\}$. However, the next proposition shows that in fact this cannot happen.

(4.2) Proposition. For every flag $(c, A)$ the group $\Gamma_{[c, A]}^{s}$ is a closed subgroup of $\Gamma$.

Proof. If $\operatorname{dim} \mathscr{S} \leq 4$, then $\Gamma_{[c, A]}^{s}=\Gamma_{[c, A]}$ according to Löwen, [26], (3.4), and the assertion is obvious. Hence we may assume that $\operatorname{dim} \mathscr{S} \geq 8$. Let $\gamma \in \Gamma_{[c, A]}^{s}$. By definition, the collineation $\gamma$ fixes some neighborhood $O$ of $c$ in $A$ pointwise. By [26], (3.3) every element of $\Gamma_{[c, A]}^{s}$ fixes some connected component of $A \backslash\{c\}$ pointwise. We will show that there is a common connected component which is fixed pointwise by any $\gamma \in \Gamma_{[c, A]}^{s}$. Being a manifold, the line $A$ is locally connected as well as a Cantor manifold, i.e. any connected component of $A$ cannot be separated by a set of dimension at most $\operatorname{dim} A-2, \mathrm{cp}$. Löwen, [28], Theorem 11. Thus we may choose $O$ to be connected, and since $\operatorname{dim} O=\operatorname{dim} A \geq 4$, we infer that $O \backslash\{c\}$ is still con- 
nected. Let $C$ denote the connected component of $A \backslash\{c\}$ containing $O \backslash\{c\}$. Since every element of $\Gamma_{[c, A]}^{s}$ fixes $C \cup\{c\}$ pointwise, the assertion follows.

The impact of the last result on the maximal compact subgroups of $\Gamma_{[c, A]}^{s}$ is as follows.

(4.3) Corollary. If $(c, A)$ is a flag, then $\Gamma_{[c, A]}^{s}$ does not contain any non-trivial compact subgroup.

Proof. Since $\Gamma_{[c, A]}^{s}$ is closed in $\Gamma$ by Proposition (4.2), the group $\Gamma_{[c, A]}^{s}$ is a Lie group. A non-trivial compact subgroup $\mathrm{K}$ of a Lie group is either finite or contains a torus subgroup. In either case, such a group $K$ contains non-trivial elements of finite order if $\mathrm{K} \neq 1$. But $\Gamma_{[c, A]}^{s}$ does not contain any non-trivial element of finite order, see Löwen, [26], (3.2). Hence the claim follows.

By Löwen, [26], (3.18) we obtain another important consequence.

(4.4) Corollary. If $\Gamma_{[c, A]}^{s} \neq 1$ for some flag $(c, A)$, then every line of $\mathscr{S}$ different from $A$ is homeomorphic to $\mathbb{R}^{l}$ or to $\mathbb{S}_{l}$. Moreover, we have $P \backslash A \approx \mathbb{R}^{2 l}$, and every line of the smooth stable plane $(P \backslash A, \mathscr{L} \backslash\{A\})$ is diffeomorphic to $\mathbb{R}^{l}$.

The next lemma collects the results of Propositions (3.2), (3.3) and (3.4) of Löwen's paper [26]. Note that for smooth stable planes we need not assume that $\operatorname{dim} \mathscr{S} \leq 4$. In order to formulate the next lemma we need the notion of coaffine points. A point $p \in P$ is called coaffine if and only if exactly one line $L$ of $\mathscr{L}_{p}$ is not compact. In this case, the line $L$ is called pointwise coaffine, see [26], (1.4) and (1.6).

(4.5) Lemma. Let $\gamma \in \Gamma_{[c, A]}^{s} \backslash\{1\}$ for some flag $(c, A)$. Then the following statements hold:

a) For every point $x \in P \backslash A$, the iterated images $x^{\gamma^{n}}$ converge to $c$ as $n \rightarrow \infty$.

b) The smooth stable plane $\mathscr{S}$ is either projective, or the line $A$ is pointwise coaffine.

c) If $\gamma$ fixes some point $x \in P \backslash A$, then $\gamma$ is the identity.

Proof. In order to prove part a), we assume for a start that $\left(x^{\gamma^{n}}\right)_{n \in \mathbb{N}}$ has an accumulation point $y \in P \backslash\{c\}$. Then there is a collineation $\delta$ which is a cluster point of $\left\{\gamma^{n}\right\}_{n \in \mathbb{N}}$ with respect to the compact-open topology such that $x^{\delta}=y$, see [26], Prop. 3.8. By Weil's lemma, either $\langle\gamma\rangle \leq \Gamma$ is relatively compact or $\langle\gamma\rangle$ is discrete. Because $\Gamma_{[c, A]}^{s}$ is a closed subgroup of $\Gamma$ which has no non-trivial compact subgroup (Proposition (4.2) and Corollary (4.3)), we conclude that $\langle\gamma\rangle$ is discrete with respect to the induced topology. In particular, the set $\left\{\gamma^{n}\right\}_{n \in \mathbb{N}}$ has no cluster point at all, a contradiction. Thus, it remains to verify that $\left(x^{\gamma^{n}}\right)_{n \in \mathbb{N}}$ accumulates at $c$. For that, choose some point $p \in A \backslash\{c\}$ which is fixed by $\gamma$. Since the line pencil $\mathscr{L}_{p}$ is compact by [25], (1.17), the sequence $\left(x^{\gamma^{n}} \vee p\right)_{n \in \mathbb{N}}$ has a cluster line $L \in \mathscr{L}_{p}$. For the sake of simplicity we will assume that this sequence converges to $L$ in $\mathscr{L}_{p}$. Since $x^{\gamma^{n}} \vee c=x \vee c$ for any $n \in \mathbb{N}$ (recall that $c$ is the center of $\gamma$ ), the sequence $x^{\gamma^{n}} \vee p$ 
of lines converges to $A$ if and only if the sequence $x^{\gamma^{n}}$ converges to $c$. Thus we may suppose that $L \neq A$. Fix some point $q \in L \backslash\{p\}$. By the stability axiom (S1), almost all lines $x^{\gamma^{n}} \vee p$ intersect the line $c \vee q$, and by continuity of the intersection map $\wedge$, the sequence $\left(q_{n}\right)_{n \geq k}$ of these intersection points converges to $q$. Hence, from the first part of our proof we conclude that $q=c$. This finishes part a). For part b) we follow Löwen's proof [26], (3.2): choose some point $p \in A$ which is fixed by $\gamma$. If $L \in \mathscr{L}_{c} \backslash\{A\}$ is non-compact, there is some line $K \in \mathscr{L}_{p} \backslash\{A\}$ which does not intersect $L$. By stability, however, the images $K^{\gamma^{n}}$ and $L^{\gamma^{n}}=L$ do intersect for sufficiently large $n \in \mathbb{N}$, because $K^{\nu^{n}}$ converges to $A$ by part a) and because $L$ intersects $A$. Thus, the lines $K$ and $L$ intersect, too. This contradiction shows that the only possible non-compact line of $\mathscr{L}_{c}$ is the line $A$. If $A$ is compact, then the smooth projective plane $\mathscr{S}$ is projective by [26], (1.3). If, on the other hand, the line $A$ is not compact, then $A$ is pointwise coaffine, see [26], (1.6). This proves part b). Part c) is an immediate consequence of a).

Using part b) of the proposition above and the remark before (3.5) in [26], we get the following important corollary.

(4.6) Corollary. Every semi-elation is an elation, i.e. $\Gamma_{[c, A]}^{s}=\Gamma_{[c, A]}$ for every flag $(c, A)$. In particular, we have $\Gamma_{[c, c]}^{s}=\Gamma_{[c, c]}$ for every point $c$ and $\Gamma_{[A, A]}^{s}=\Gamma_{[A, A]}$ for every line A.

Note that in the topological (non-smooth) case there are stable planes which admit proper semi-elations, see Löwe, [24], Example 6. Hence, Löwe's example of a shear plane is a non-smooth stable plane, i.e. there are no smooth structures on $P$ and $\mathscr{L}$ which turn $\mathscr{S}=(P, \mathscr{L})$ into a smooth stable plane.

We note several more consequences of Lemma (4.5), part b). The first one is based on the characterization of pointwise affine lines (for a definition see Löwen [26], (1.6)), while the second one follows from [26], (3.5).

(4.7) Corollary. If $\Gamma_{[c, A]}^{s} \neq 1$ for some flag $(c, A)$, then the smooth stable plane $\mathscr{S}$ has a compact line.

(4.8) Corollary. If $\Gamma_{[c, A]}^{s} \neq 1$ for some flag $(c, A)$, then every central collineation has an axis.

(4.9) Corollary. For every point $c \in P$ the group $\Gamma_{[c, c]}$ can be written as the union $\Gamma_{[c, c]}=\bigcup_{A \in \mathscr{L}_{c}} \Gamma_{[c, A]}$.

Proof. Let $c \in P$. If $\Gamma_{[c, A]}=\mathbb{1}$ for every line $A \in \mathscr{L}_{c}$ or if $\mathscr{S}$ is a projective plane, nothing remains to be proved. Thus, by Corollary (4.8), every element of $\Gamma_{[c, c]}$ has an axis and it suffices to verify that the product of two elations in $\Gamma_{[c, c]}$ is an elation again. So let $\alpha \in \Gamma_{[c, A]}$ and $\beta \in \Gamma_{\left[c, A^{\prime}\right]}$, where $A \neq A^{\prime}$, and denote by $L$ the axis of the product $\alpha \beta$. By Lemma (4.5), part b), the lines $A$ and $A^{\prime}$ are pointwise coaffine. In particular, the point $c$ is coaffine and thus at least one of the two lines $A$ and $A^{\prime}$ 
has to be compact (see Löwen [26], (1.6)). Let $A$ be a compact line. Then $A$ meets $L$ in some point $p$ ([25], (1.15)) and from $p^{\beta}=p^{\alpha \beta}=p$ together with Lemma (4.5), part b), we conclude that $p=A \wedge A^{\prime}=c$. Thus the axis $L$ of $\alpha \beta$ goes through $c$ and $\alpha \beta$ is an elation with center $c$.

According to Löwen [26], (3.2), the last corollary implies that $\Gamma_{[c, c]}$ contains no nontrivial element of finite order. This result is false for (topological) stable planes. Löwe has given an example of a shear plane ([24], Example 6) which admits a group of semi-elations isomorphic to the compact group $\operatorname{Spin}_{3} \mathbb{R}$.

(4.10) Corollary. For every point $c \in P$ the elation group $\Gamma_{[c, c]}$ is torsion-free.

Our proof of the preceding corollary mainly relies on the fact that a central collineation has an axis in the presence of compact lines. Unfortunately, the dual of this is not true. Thus we are forced to look for another way of proving the dual of Corollary (4.10). We divide this proof into two separate parts.

(4.11) Proposition. Let $(c, A),\left(c^{\prime}, A\right)$ and $\left(c, A^{\prime}\right)$ be flags. If $\gamma \in \Gamma_{[c, A]}$ and $\gamma^{\prime} \in \Gamma_{\left[c^{\prime}, A\right]} \cup \Gamma_{\left[c, A^{\prime}\right]}$, then either $\gamma \gamma^{\prime}=1$ or $\gamma \gamma^{\prime}$ has infinite order.

Proof. Let $\gamma^{\prime} \in \Gamma_{\left[c, A^{\prime}\right]}$. If $A=A^{\prime}$, then the claim follows from [26], (3.2). So let us suppose that $A \neq A^{\prime}$ and that $\left(\gamma \gamma^{\prime}\right)^{k}=\mathbb{1}$ holds for some integer $k$. Since $A \neq A^{\prime}$, the collineations $\gamma$ and $\gamma^{\prime}$ commute by Lemma (4.1), and we get $\mathbb{1}=\left(\gamma \gamma^{\prime}\right)^{k}=\gamma^{k} \gamma^{\prime k}$. Note also that in this case we have $\gamma \gamma^{\prime} \neq 1$. We conclude that $\gamma^{k} \in \Gamma_{[c, A]} \cap \Gamma_{\left[c, A^{\prime}\right]}$. By [25], (3.4), this intersection is just the trivial group, and thus $k=0$ follows by Corollary (4.3). If $\gamma^{\prime} \in \Gamma_{\left[c^{\prime}, A\right]}$, the assertion is proved analoguously, for Lemma (4.1) is self-dual and the dual statement of [25], (3.4) is trivially true.

(4.12) Theorem. Every non-trivial element of $\Gamma_{[A, A]}$ has infinite order.

Proof. Let $\gamma \in \Gamma_{[A, A]} \backslash\{1\}$. Because $\Gamma_{[A, A]}$ is generated by the groups $\Gamma_{[c, A]}$ for $c \in A$, the element $\gamma$ can be written as a product $\gamma=\gamma_{1} \cdots \gamma_{n}$ with $\gamma_{i} \in \Gamma_{\left[c_{i}, A\right]}$ for some points $c_{i} \in A$. If $n=2$, the assertion follows from Proposition (4.11). Hence, by induction on $n$ we may assume that $\gamma=\delta \gamma_{n}$, where $\delta \in \Gamma_{[A, A]}$ has infinite order and does not act trivially on the line pencil $\mathscr{L}_{c_{n}}$. Suppose that $\left(\delta \gamma_{n}\right)^{k}=1$ for some integer $k$. In view of Lemma (4.1) and Proposition (4.2), we may assume that $\Gamma_{[A, A]}$ is commutative. Hence we get $\delta^{k}=\gamma_{n}^{-k} \in \Gamma_{\left[c_{n}, A\right]}$. Put $\varepsilon:=\delta^{k}$, choose some line $L \in \mathscr{L}_{c_{n}}$ with $L^{\delta} \neq L$, and select some point $x \in L \backslash\left\{c_{n}\right\}$. Since the sequence $\left(x^{\varepsilon^{m}}\right)_{m \in \mathbb{N}}$ converges to $c_{n}$ by Proposition (4.5), we infer from the stability axiom (S1) that the lines $x^{\varepsilon^{m}} \vee x^{\delta}$ meet the axis $A$ for sufficiently large integers $m$. Hence, let $p:=\left(x^{\varepsilon^{m}} \vee x^{\delta}\right) \wedge A$ for some suitable integer $m$. From $L \neq L^{\delta}$ we obtain that $p \neq c_{n}$. Because the line $x^{\varepsilon^{m}} \vee x^{\delta}$ meets the axis $A$, the equation

$$
\left(x^{\varepsilon^{m}} \vee x^{\delta}\right) \wedge A=p=p^{\delta-1}=\left(x^{\varepsilon^{m} \delta^{-1}} \vee x\right) \wedge A
$$

shows that $K:=x^{\varepsilon^{m} \delta^{-1}} \vee x$ is a fixed line of $\varepsilon^{m} \delta^{-1}$. 


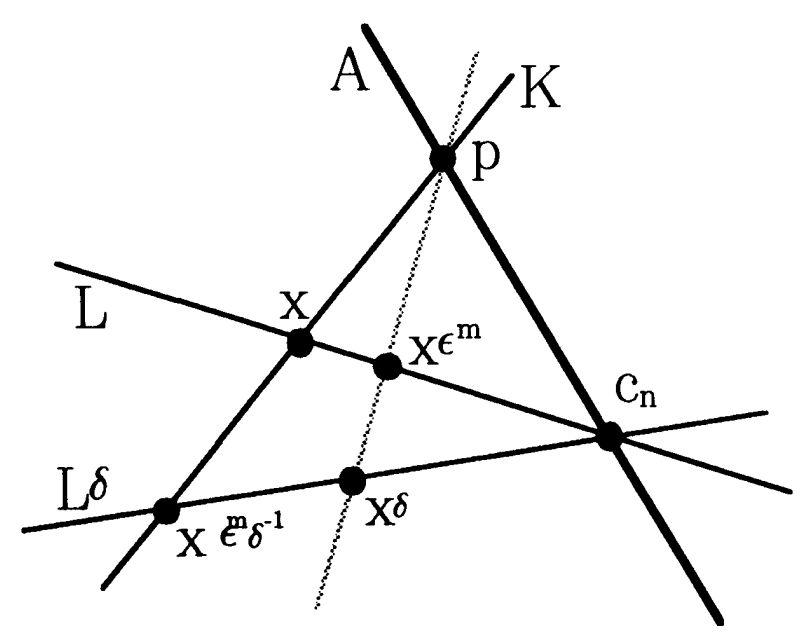

From

$$
x^{\left(\varepsilon^{m} \delta^{-1}\right)^{k i}}=x^{\varepsilon^{m k i} \delta-k i}=x^{\varepsilon^{i(m k-1)}} \quad(i \in \mathbb{N})
$$

we conclude that the sequence $\left(x^{\left(\varepsilon^{m} \delta^{-1}\right)^{k i}}\right)_{i \in \mathbb{N}}$ converges to $c_{n}$. But, on the other hand, the points $x^{\left(\varepsilon^{m} \delta^{-1}\right)^{k i}}$ lie on the fixed line $K$ which shows that this sequence cannot converge to $c_{n}$ since $c_{n} \notin K$ and $K$ is a closed subset of $P$, a contradiction. Hence ord $(\gamma)=\infty$ and the theorem is proved.

We proceed with a generalization of Corollary (3.9) by determining the kernels of the derivation maps $D_{p}$ in the stable case.

(4.13) Theorem. For every point $c$ and every line $A$ we have $\operatorname{ker} \mathrm{D}_{c}=\Gamma_{[c, c]}$, and $\operatorname{ker} \mathrm{D}_{A}$ has no non-trivial compact subgroup.

Proof. Let $c \in P$. By corollaries (3.8) and (4.6) we only have to show that $\operatorname{ker} \mathrm{D}_{c} \subseteq \Gamma_{[c, c]}$. The kernel of $\mathrm{D}_{c}$ consists of central collineations with center $c$, see Theorem (3.6). If ker $\mathrm{D}_{c}$ is not compact (e.g. if $\Gamma_{[c, c]} \neq 1$, Corollary (4.10)) then every central collineation has an axis as in the projective case, see Löwen [26], (3.5) and Corollary (4.8), and the claim follows from Theorem (3.6). Hence we may suppose that ker $\mathrm{D}_{c}$ is compact. Providing the manifold $P$ of points with a suitable Riemannian metric $\rho$ such that $\operatorname{ker}_{\mathrm{c}}$ becomes a group of isometries with respect to $\rho$ (see Bredon [6], Chap. VI, (2.1)) this shows that $\operatorname{ker} \mathrm{D}_{c}=\mathbb{1}$ (Helgason [19], Chap. I, (11.2)), and hence the inclusion $\operatorname{ker} D_{c} \subseteq \Gamma_{[c, c]}$ holds trivially. Thus we have proved that $\operatorname{ker} D_{c}=\Gamma_{[c, c]}$. Moreover, the preceding argumentation shows that a maximal compact subgroup of $\operatorname{ker} \mathrm{D}_{A}$ has to be trivial.

Since the kernel of a Lie group homomorphism is closed, the following corollary is a by-product of the last theorem. 
(4.14) Corollary. For any point $c$ the group $\Gamma_{[c, c]}$ is a closed subgroup of $\Gamma$.

It is not clear, whether or not $\Gamma_{[A, A]}$ for some line $A$ is a closed subgroup of $\Gamma$. However, the next result shows that at least the connected component $\Gamma_{[A, A]}^{1}$ is closed.

(4.15) Proposition. For every line $A$ the connected component $\Gamma_{[A, A]}^{1}$ of the elation group $\Gamma_{[A, A]}$ is a closed subgroup of $\Gamma$.

Proof. Put $\mathrm{Y}:=\left(\Gamma_{[A, A]}\right)^{1}$. If $\mathrm{Y} \neq \overline{\mathrm{Y}}$ then there is a non-closed one-parameter subgroup $P$ of $Y$, see [20], Chap. XVI, Theorem 2.4. By Stroppel, [38], (8), the Lie group $\Gamma_{[A]}$ (note that $\Gamma_{[A]}$ is of course a closed subgroup of $\Gamma$ ) does not contain distinct commuting involutions, whence the dimension of a maximal torus subgroup of $\Gamma_{[A]}$ is at most 1. This contradicts Lemma (3.13) and we have finished the proof.

So far we have proved that $\Gamma_{[c, c]}^{1}$ and $\Gamma_{[A, A]}^{1}$ are simply connected Lie groups without non-trivial compact subgroups. Decomposing $\Gamma_{[c, c]}^{1}$, say, into its solvable radical and a semi-simple Levi complement $\Psi$, this implies that $\Psi$ is either trivial or isomorphic to a direct product of quasi-simple factors each of which is isomorphic to the universal covering group $\Omega$ of $\mathrm{SL}_{2} \mathbb{R}$. In order to show that $\Gamma_{[c, c]}^{1}$ is solvable, it remains to exclude the case that $\Gamma_{[c, c]}^{1}$ contains a subgroup isomorphic to $\Omega$. In fact, we prove a more general result:

(4.16) Proposition. If $\Gamma$ contains a non-linear subgroup $\Omega$, then $\Omega_{[c, c]} \neq \Omega$ for any point $c \in P$ and dually $\Omega_{[A, A]} \neq \Omega$ for any line $A \in \mathscr{L}$.

Proof. Assume that there is a point $c \in P$ such that $\Omega_{[c, c]}=\Omega$. Since $\Omega$ is not linear, it is not abelian and Lemma (4.1) shows that $\Omega_{[c, c]}=\Omega_{[c, A]}$ for some line $A \in \mathscr{L}_{c}$. Let $p \in A \backslash\{c\}$. Because $\Omega$ contains no elation with center $p$, the mapping $\mathrm{D}_{p}: \Omega \rightarrow \mathrm{GL}\left(\mathrm{T}_{p} P\right)$ is a (continuous) injection (see Theorem (3.6) and Proposition (3.7)) which is a contradiction. Hence, we have $\Omega_{[c, c]} \neq \Omega$. The proof of the dual statement runs along the same lines.

Since the universal covering group of $\mathrm{SL}_{2} \mathbb{R}$ is not a linear group, we may summarize the information on the groups $\Gamma_{[c, c]}^{1}$ and $\Gamma_{[A, A]}^{1}$ as follows.

(4.17) Corollary. The elation groups $\Gamma_{[\mathrm{C}, \mathrm{c}]}^{1}$ and $\Gamma_{[\mathrm{A}, \mathrm{A}]}^{1}$ are closed simply connected solvable subgroups of $\Gamma$. In particular, these groups are linear groups.

Using the fact that the tangent translation plane $\mathscr{A}_{c}$ at the point $c$ is an affine translation plane (this is, in general, not true for the tangent object $\mathscr{A}_{L}$ of some line $L$, see [4], section 4), we can even prove the following result.

(4.18) Theorem. For any point $c \in P$ the elation group $\Gamma_{[c, c]}$ is abelian and $\Gamma_{[c, c]}^{1} \cong \mathbb{R}^{m}$ with $m \leq n$. 
Proof. Let $A \in \mathscr{L}_{c}$ und choose some point $p \in A \backslash\{c\}$. The derivation map

$$
D_{p}: \Gamma_{[c, A]} \rightarrow Y \leq \operatorname{Aut}\left(\mathscr{A}_{p}\right)
$$

is injective and its image is contained in the group $Y$ of all shears of $\mathscr{A}_{p}$ with axis $\mathrm{T}_{p} A$. The group $\mathrm{Y}$ is commutative, because $\mathscr{A}_{p}$ is a translation plane. Moreover, since every quasifield that coordinatizes this plane has an additive group which is isomorphic to $\mathbb{R}^{l}$, and because $Y$ can be regarded as a subgroup of such a quasifield, we have $Y \leq \mathbb{R}^{l}$, see [35], (44.8(b)) and Section 25. If $\Gamma_{[c, c]}=\Gamma_{[c, A]}$, then there is nothing left to prove. Hence, we may assume that $\Gamma_{[c, c]}$ contains two elations with different axes. Then $\Gamma_{[c, c]}$ is commutative by Lemma (4.1). Since this group is torsionfree according to (4.10), its connected component $\Gamma_{[c, c]}^{1}$ is isomorphic to $\mathbb{R}^{m}$ for some $m \geq 0$. The group $\Gamma_{[c, c]}^{1}$ acts on $\mathscr{L} \backslash \mathscr{L}_{c}$ with trivial stabilizers. Hence, the dimension formula gives $m=\operatorname{dim} \Gamma_{[c, c]}^{1} \leq \operatorname{dim} \mathscr{L} \backslash \mathscr{L}_{c}=n$ and the theorem is proved.

In the next theorem we will use the well-known formula $\operatorname{dim} G=\operatorname{dim} H+\operatorname{dim} N$ for a Lie group $G$, a closed subgroup $N$ of $G$ and $H=G / N$.

(4.19) Theorem. If $\Delta$ is a closed connected solvable subgroup of $\Gamma$ which fixes some point or some line, then $\Delta$ is a linear Lie group.

Proof. The group $\Delta$ is a Lie group, since it is a closed subgroup of the Lie group $\Gamma$. Assume that $\Delta$ fixes some point $p$. Consider the derivation map $\mathrm{D}_{p}: \Delta \rightarrow \operatorname{Aut}\left(\mathscr{A}_{p}\right)$. By the last two results the connected component $\Lambda$ of $\operatorname{ker} D_{p}$ is a simply connected solvable group. Of course, the quotient group $\Delta / \operatorname{ker} \mathrm{D}_{p}$ is a solvable linear group. Hence, the quotient $Y=\Delta / \Lambda$ is also a solvable linear group according to [32], 5.5(2). By a Theorem of Malcev (see [30] or [32], 7.1), the quotient $Y$ can be decomposed as $Y=T \ltimes N$, where $T$ is a maximal compact subgroup of $Y$ and $N$ is simply connected. We denote by $\pi: \Delta \rightarrow Y$ the canonical projection of $\Delta$ onto the quotient $Y$. Consider the normal subgroup $M=\pi^{-1}(N)$ of $\Delta$. The connected component $M^{1}$ is simply connected, otherwise it contains a torus subgroup $P$. Because $N$ is simply connected and solvable, we infer that $\pi(P)=1$, whence $P \leq \operatorname{ker} \pi=\Lambda$, a contradiction to the fact that $\Lambda$ is simply connected and solvable. Moreover, we have $\operatorname{dim} M=\operatorname{dim} N+\operatorname{dim} \Lambda$. Let $K$ denote a maximal compact subgroup of $\Delta$. The group $K$ is a torus group which has trivial intersection with $M^{1}$. Moreover, we have $\operatorname{dim} K=\operatorname{dim} T$, because the kernel $\Lambda$ is connected and contains no torus subgroup, and we get

$$
\begin{aligned}
\operatorname{dim} \Delta & =\operatorname{dim} Y+\operatorname{dim} \Lambda=\operatorname{dim} T+\operatorname{dim} N+\operatorname{dim} \Lambda=\operatorname{dim} T+\operatorname{dim} M \\
& \geq \operatorname{dim} K+\operatorname{dim} M .
\end{aligned}
$$

Since $\Delta$ and $\mathrm{K}$ are connected, this implies that $\mathrm{K}$ is a complement of $\mathrm{M}^{1}$ in $\Delta$ and we can write $\Delta$ as the semi-direct product $\Delta=\mathrm{K} \ltimes \mathrm{M}^{1}$. By [32], 7.1, this shows 
that $\Delta$ is a linear group. The same argument can be used in order to prove the dual statement.

Since for compact projective planes any solvable subgroup has some fixed point or some fixed line (see [29], VII, Satz 3), the last theorem yields.

(4.20) Corollary. If $\Delta$ is a closed connected solvable subgroup of $\Gamma$ and if $\mathscr{S}$ is a smooth projective plane, then $\Delta$ is a linear Lie group.

\section{Reflections}

In the last section we study reflections $\alpha$ of a smooth stable plane $\mathscr{S}$, i.e. $\alpha$ is an involutory central or axial collineation of $\mathscr{P}$. Recall that we do not have to assume that $\alpha$ is continuous or even smooth, since this is always true for central or axial collineations, see the introduction to Section 3. Moreover, we do not suppose that a reflection $\alpha \in \Gamma_{[c]}$ has an axis or, dually, that a reflection $\beta \in \Gamma_{[A]}$ has a center.

We are going to transfer the results of Löwen, [26], p. 303-306 to the smooth case. As in the previous section it turns out that these results hold without the restriction to low dimensions. Before we start with the dual statement of Stroppel, [38], (8), we prove a lemma about the eigenspaces of linearized involutions.

(5.1) Lemma. For $x \in P \cup \mathscr{L}$ let $\alpha \in \Gamma_{x}$ be an involution. If $\Xi_{+}$and $\Xi_{-}$denote the positive and negative eigenspaces of $\mathrm{D}_{x} \alpha$, respectively, then either $\operatorname{dim} \Xi_{+}=\operatorname{dim} \Xi_{-}$ $=\frac{1}{2} \operatorname{dim} P=l$ or $x$ is an axis (if $x \in \mathscr{L}$ ) or a center (if $x \in P$ ) of $\alpha$.

Proof. Let $x \in \mathscr{L}$. By Theorem (4.13) we have $\mathrm{D}_{x} \alpha \neq 1$. If $\operatorname{dim} \Xi_{+}>l$, then the intersection $\mathrm{T}_{x} \mathscr{L}_{p} \cap \Xi_{+}$is not trivial for every point $p \in x$. Now, $\mathrm{D}_{x} \alpha$ acts on the set of tangent spaces $\mathrm{T}_{x} \mathscr{L}_{p}$ and so $\mathrm{D}_{L} \alpha$ leaves invariant every subspace $\mathrm{T}_{x} \mathscr{L}_{p}$. The same argument shows that $\mathrm{D}_{x} \alpha$ acts trivially on the partial spread $\left\{\mathrm{T}_{x} \mathscr{L}_{p} \mid p \in x\right\}$ of tangent spaces if $\operatorname{dim} \equiv_{-}>l$. This, however, implies that in both cases $x$ is an axis of $\alpha$. Being an involution, the map $D_{x} \alpha$ is diagonalizable with \pm 1 as its only eigenvalues. Hence we conclude that $\operatorname{dim} \Xi_{+}=l=\operatorname{dim} \Xi_{-}$if $x$ is not an axis. If $x \in P$, the proof runs the same way.

(5.2) Theorem. The group $\Gamma_{[c]}$ contains no pair of commuting reflections.

Proof. Let $\alpha, \beta \in \Gamma_{[c]}$ be two different commuting reflections. Then none of the collineations $\alpha, \beta$ and $\alpha \beta$ is an elation by Corollary (4.3). If $\alpha$ fixes some point $p \neq c$, then $\alpha$ has an axis $A \in \mathscr{L}_{p}$ with $c \notin A$ ([26], (3.3)). Since $\alpha$ and $\beta$ commute, this means that $\mathrm{E}:=\langle\alpha, \beta\rangle$ is a subgroup of $\mathrm{\Gamma}_{[c, A]}$. Thus the group $\mathrm{E}$ acts freely on $\mathscr{L}_{p} \backslash\{A, p \vee c\}$, see Löwen [25], (3.4). By a theorem of P. A. Smith [37], p. 407, we conclude that $\mathrm{E}$ has periodic cohomology and hence must be a cyclic group ([11], p. 262), a contradiction. Thus, for the rest of the proof we may assume that for some fixed line $L \in \mathscr{L}_{c}$ the group E acts freely on $L \backslash\{c\}$. In particular, we infer that 
the derivation map $\mathrm{D}_{L}: \mathrm{E} \rightarrow \mathrm{GL}\left(\mathrm{T}_{L} \mathscr{L}\right)$ is injective. Since $\alpha$ and $\beta$ act trivially on the submanifold $\mathscr{L}_{c}$, their derivations $\mathrm{D}_{L} \alpha$ and $\mathrm{D}_{L} \beta$ both induce the identity on the tangent space $\mathrm{T}_{L} \mathscr{L}_{c}$. According to Lemma (5.1) we may thus write

$$
\mathrm{D}_{L} \alpha=\left(\begin{array}{cc}
-1 & \\
& 1
\end{array}\right) \text { and } \mathrm{D}_{L} \beta=\left(\begin{array}{cc}
X & \mathrm{Y} \\
& 1
\end{array}\right)
$$

with respect to a suitable basis of $\mathrm{T}_{L} \mathscr{L}$, where all entries are $l$-dimensional square matrices. Since $\mathrm{D}_{L} \alpha$ and $\mathrm{D}_{L} \beta$ commute, we get $Y=0$, and since the negative eigenspace of $\beta$ has dimension $l$, we obtain $X=-1$. Hence, we have $\mathrm{D}_{L} \alpha=\mathrm{D}_{L} \beta$ and thus $\mathrm{D}_{L}(\alpha \beta)=1$, which is a contradiction to the injectivity of $\mathrm{D}_{L}$ on $\mathrm{E}$.

Now we turn to Löwen's results on reflections which he verified for stable planes of dimensions at most 4 . Note that no proofs of the following two results exist even in the case of compact projective planes.

(5.3) Lemma. If $\alpha, \beta \in \Gamma_{[c]}$ are different reflections, then $\alpha \beta$ is an elation. In particular, the smooth stable plane $\mathscr{S}$ is either projective or the axis of $\alpha \beta$ is pointwise coaffine.

Proof. Let $\alpha, \beta \in \Gamma_{[c]}$ be two different reflections and set $\mathrm{E}:=\overline{\langle\alpha, \beta\rangle}=\overline{\langle\alpha \beta\rangle} \cdot\langle\alpha\rangle$. Fix some line $L \in \mathscr{L}_{c}$. Assume that $\alpha \beta$ acts non-trivially on $L$. Then $\alpha$ and $\beta$ induce on $L$ different involutions. Denoting by $\mathrm{N}$ the kernel of the action of $\mathrm{E}$ on $L$, we put $\Phi:=\overline{\langle\alpha \beta\rangle} /(\mathrm{N} \cap \overline{\langle\alpha \beta\rangle})$.

Now we are going to prove that $\Phi$ is infinite. As in the proof of Theorem (5.2), we may choose a basis of $\mathrm{T}_{L} \mathscr{L}$ such that

$$
\mathrm{D}_{L} \alpha=\left(\begin{array}{cc}
-1 & \\
& 1
\end{array}\right) \text { and } \mathrm{D}_{L} \beta=\left(\begin{array}{cc}
X & Y \\
& 1
\end{array}\right)
$$

From $\left(\mathrm{D}_{L} \beta\right)^{2}=1$ we infer that $X^{2}=1$ and $(X+1) Y=0$. Setting $M=\left(\mathrm{D}_{L} \alpha\right)\left(\mathrm{D}_{L} \beta\right)$ we get

$$
M^{n}=\left(\begin{array}{cl}
(-X)^{n} & \left.\left(\left[\frac{n+1}{2}\right]-\left[\frac{n}{2}\right] X\right) Y\right) . \\
\mathbb{1}
\end{array}\right)
$$

If $M^{n}=1$, then $n$ must be even and thus

$$
M^{n}=\left(\begin{array}{ll}
1 & \frac{n}{2}(1-X) Y \\
1
\end{array}\right)=\mathbb{1}
$$


holds. Hence we have $(1-X) Y=0$. Combining this with $(1+X) Y=0$ we get $Y=0$. But this implies that $\mathrm{D}_{L} \alpha$ and $\mathrm{D}_{L} \beta$ commute and we obtain $\mathrm{D}_{L} \alpha=\mathrm{D}_{L} \beta$ as in the proof of Theorem (5.2). We conclude that $\alpha \beta$ acts trivially on $L$ which is a contradiction. Hence the group $\Phi$ is infinite.

By Weil's lemma (cp. [20], Chapt. 16, (2.3)) the group $\Phi$ is either discrete or compact. If $\Phi$ is discrete, the orbits of $\langle\alpha \beta\rangle$ on $L$ have at most two ends. One of these ends is the center $c$, while the other end is either $\infty$ or it lies on a semi-axis of $\alpha \beta$, see [26], (3.8) and (3.9). By conjugation, the reflection $\alpha$ induces inversion on $\langle\alpha \beta\rangle$, and thus $\alpha$ interchanges the ends of the orbits in question. Since $\alpha$ fixes the point $c$, both ends most coincide. In particular, the point $c$ must lie on a semi-axis $A$ of $\alpha \beta$. By Lemma (4.5), the line $A$ is pointwise coaffine and $\alpha \beta$ is an elation by Corollary (4.6). If, on the other hand, $\Phi$ is compact, it contains a maximal torus subgroup $\mathrm{T}$ of positive dimension $t$ (recall that $\Phi$ is infinite). If $t=1$, then $\alpha$ centralizes the unique involution $\omega$ of T (note that $\alpha$ normalizes $\overline{\langle\alpha \beta\rangle}$ ). Since $\alpha$ does not commute with $\beta$ by Theorem (5.2), we have $\alpha \neq \omega$. If $t>1$, the group T possesses a pair of commuting involutions as well. But according to Theorem (5.2), the group $\Gamma_{[c]}$ contains no commuting pair of involutions. Thus, the group $\Phi$ cannot be compact and the lemma is proved.

As an immediate consequence of the last lemma we obtain the following result which can also be deduced in a less direct way from corollaries (3.14) and (4.3).

(5.4) Corollary. For every pair $(c, A) \in P \times \mathscr{L}$ the group $\Gamma_{[c, A]}$ contains at most one reflection.

The last two results transfer Proposition 3.22 and Corollary 3.23 of Löwen [26] to smooth stable planes.

(5.5) Proposition. Let $\alpha \in \Gamma_{[c, A]}$ and $\beta \in \Gamma_{[a]}$ be reflections, where $a \in A$. Then either $(\alpha \beta)^{2} \in \Gamma_{[a, A]} \backslash\{\mathbb{1}\}$ and $A$ is pointwise coaffine, or $\alpha \beta$ is an involution with axis $a \vee c$, and $\beta$ has an axis $B \in \mathscr{L}_{c}$.

Proof. Put $L:=a \vee c$. Clearly, the product $(\alpha \beta)^{2}$ is an element of $\Gamma_{[a, A]}$. If $(\alpha \beta)^{2} \neq 1$, the assertion follows from Löwen [26], (3.2) and Lemma (4.5). Hence, let $\alpha \beta$ be an involution. Since $A \neq L$ is the (unique) axis of $\alpha$, we get the representation $\mathrm{D}_{L} \alpha=\left({ }^{-1}{ }_{1}\right)$ from Lemma (5.1). Because $\alpha$ fixes every line through $c$ and acts nontrivially on $\mathscr{L}_{a}$, we have $\left.\mathrm{D}_{L} \alpha\right|_{\mathscr{L}_{c}}=\mathbb{1}$ and $\left.\mathrm{D}_{L} \alpha\right|_{\mathscr{L}_{a}}=-1$. Moreover, we know that $\left.\mathrm{D}_{L} \beta\right|_{\mathscr{L}_{a}}=1$, since $a$ is the center of $\beta$. This implies that $\mathrm{D}_{L} \beta=\left(\begin{array}{ll}1 & Y \\ X\end{array}\right)$ with respect to the decomposition $\mathrm{T}_{L} \mathscr{L}=\mathrm{T}_{L} \mathscr{L}_{a} \oplus \mathrm{T}_{L} \mathscr{L}_{c}$. Recalling that $\alpha$ and $\beta$ commute, we infer that $Y=0$. The line $L$ cannot be an axis of $\beta$, for $\beta$ is a reflection. Consequently, we derive from Lemma (5.1) that $X=-1$ and hence $D_{L}(\alpha \beta)=-$ id follows. Clearly, this implies that $L$ is an axis of $\alpha \beta$. The last claim follows from Lemma (4.5), part c), because $\beta$ fixes the point $c$.

As in Löwen's paper [26], the last result implies the following corollary. 
(5.6) Corollary. Let $\alpha \in \Gamma_{[a, A]}$ and $\beta \in \Gamma_{[b, B]}$ be reflections, where $a \in B$ and $b \in A$. Then $\alpha \beta$ is a reflection with axis $a \vee b$.

\section{References}

[1] Baer, R.: Projectivities with fixed points on every line of the plane. Bull. Amer. Math. Soc. 52 (1946), 273-286

[2] Betten, D.: 2-dimensionale differenzierbare projektive Ebenen. Arch. Math. 22 (1971), 304-309

[3] Bödi, R.: Automorphism groups of differentiable double loops. Geom. Dedicata 46 (1993), 61-72

[4] Bödi, R.: Smooth stable planes. Results Math. 31 (1997), 300-321

[5] Bödi, R., Kramer, L.: Differentiability of continuous homomorphisms between smooth loops. Results Math. 25 (1994), 13-19

[6] Bredon, G. E.: Introduction to Compact Transformation Groups. New York, London: Academic Press 1972

[7] Breitsprecher, S.: Einzigkeit der reellen und der komplexen projektiven Ebene. Math. Z. 99 (1967), 429-432

[8] Breitsprecher, S.: Projektive Ebenen, die Mannigfaltigkeiten sind. Math. Z. 121 (1971), 157-174

[9] Breitsprecher, S.: Zur topologischen Struktur zweidimensionaler projektiver Ebenen. Geom. Dedicata 1 (1972), 21-32

[10] Buchanan, T., Hähl, H.: On the kernel and the nuclei of 8-dimensional locally compact quasifields. Arch. Math. 29 (1977), 472-480

[11] Cartan, H., Eilenberg, S.: Homological Algebra. Princeton: Princeton University Press 1956

[12] Floyd, E. E.: On periodic maps and the Euler characteristic of associated spaces. Trans. Amer. Math. Soc. 72 (1952), 138-147

[13] Floyd, E. E.: Orbits of torus groups acting on manifolds. Ann. of Math. 65 (1957), $505-512$

[14] Grundhöfer, T., Hähl, H.: Fibrations of spheres by great spheres over division algebras and their differentiability. J. Differential Geometry 31 (1990), 357-363

[15] Hähl, H.: Automorphismengruppen von lokalkompakten zusammenhängenden Quasikörpern und Translationsebenen. Geom. Dedicata 4 (1975), 305-321

[16] Hähl, H.: Lokalkompakte zusammenhängende Translationsgruppen mit großen Sphärenbahnen auf der Translationsachse. Results Math. 2 (1979), 62-87

[17] Hähl, H.: Homologies and elations in compact, connected projective planes. Topology Appl. 12 (1981), 49-63

[18] Hähl, H.: Differentiable fibrations of the $(2 n-1)$-sphere by great $(n-1)$-spheres and their coordinatization over quasifields. Results Math. 12 (1987), 99-118

[19] Helgason, S.: Differential Geometry and Symmetric Spaces. New York, London: Academic Press 1962

[20] Hochschild, G.: The structure of Lie groups. San Francisco, London, Amsterdam: Holden-Day 1965

[21] Kozma, J.: On the differentiability of loopmultiplication in canonical coordinate-system. Publ. Math. Debrecen 37 (1990), 313-325 
[22] Kozma, J.: Behaviour of loops in a canonical coordinate system. Arch. Math. 55 (1990), 498-502

[23] Liao, S. D.: A theorem on periodic maps of homology spheres. Ann. of Math. 56 (1952), 68-83

[24] Löwe, H.: Shear planes. Geom. Dedicata 52 (1994), 87-104

[25] Löwen, R.: Vierdimensionale stabile Ebenen. Geom. Dedicata 5 (1976), 239-294

[26] Löwen, R.: Central collineations and the parallel axiom in stable planes. Geom. Dedicata 10 (1981), 283-315

[27] Löwen, R.: Stable planes of low dimension admitting reflections at many lines. Results Math. 5 (1982), 60-80

[28] Löwen, R.: Topology and dimension of stable planes: On a conjecture of H. Freudenthal. J. Reine Angew. Math. 343 (1983), 108-122

[29] Lüneburg, M.: Involutionen, auflösbare Gruppen und die Klassifikation topologischer Ebenen. Mitt. Math. Sem. Giessen 209 (1992), 1-113

[30] Malcev, A. I.: On piecewise connected locally closed groups. Dokl. Akad. Nauk SSSR 40 (1943), 108-110

[31] Montgomery, D., Zippin, L.: Topological transformation groups. New York: Interscience Publ., Inc. 1955

[32] Onishchik, A.L., Vinberg, E. B.: Lie Groups and Lie Algebras III. Berlin-New York: Springer 1994

[33] Otte, J.: Differenzierbare Ebenen. Dissertation Kiel 1992

[34] Otte, J.: Smooth Projective Translation Planes. Geom. Dedicata 58 (1995), 203-212

[35] Salzmann, H., Betten, D., Grundhöfer, T., Hähl, H., Löwen, R., Stroppel, M.: Compact Projective Planes. De Gruyter 1995

[36] Seidel, H.-P.: Groups of homologies in 4-dimensional stable planes are classical. Combinatories '86 (Proc. Conf. Toronto, 1986), 399-403

[37] Smith, P.A.: New Results and Old Problems in Finite Transformation Groups. Bull. Amer. Math. Soc. 66 (1960), 401-415

[38] Stroppel, M.: Quasiperspectivities in stable planes. Monatsh. Math. 115(1993), 183-189

Received February 5, 1997; revised and in final form July 8, 1997

Richard Bödi, Mathematisches Institut, Universität Tübingen, Auf der Morgenstelle 10, 72076 Tübingen, Germany

richard.boedi@uni-tuebingen.de 
Bereitgestellt von | ZHAW Zuercher Hochschule für Angewandte Wissenschaften 